\title{
Period variation studies of six contact binaries in M4
}

\author{
Jagirdar Rukmini*, Devarapalli Shanti Priya ${ }^{\dagger}$ \\ Department of Astronomy, Osmania University, Hyderabad, Telangana - 500007
}

\begin{abstract}
We present the first period study of six contact binaries in the closest globular cluster M4 the data collected from June 1995-June 2009 and Oct 2012-Sept 2013. New times of minima are determined for all the six variables and eclipse timing (O-C) diagrams along with the quadratic fit are presented. For all the variables, the study of $(\mathrm{O}-\mathrm{C})$ variations reveal changes in the periods. In addition, the fundamental parameters for four of the contact binaries obtained using the Wilson-Devinney code (v2003) are presented. Planned observations of these binaries using the 3.6-m Devasthal Optical Telescope (DOT) and the 4-m International Liquid Mirror Telescope (ILMT) operated by the Aryabhatta Research Institute of Observational Sciences (ARIES; Nainital) can throw light on their evolutionary status from long term period variation studies.
\end{abstract}

\section{Introduction}

The study of contact binaries gives insight into stellar evolution theory through fundamental stellar parameters like the mass, radius, luminosity and composition (Yakut \& Eggleton 2005). M4, also known as NGC 6121, is the closest galactic globular cluster. Binaries in globular clusters not only provide useful information on the dynamical evolution of globular clusters but also are windows to understand the absolute dimensions of globular cluster stars. Only few eclipsing binaries in globular clusters have been analyzed so far compared to field stars. In this paper, we present the first period study for six contact binaries (V47, V48, V49, V51, V53, V55) in the globular cluster M4, for the data collected between 1995-2009. Also, the photometric solution for four of the contact binaries, which were not studied before, is obtained using the Wilson-Devinney (WD) code (v2003) for the data collected between 1995-2013.

\section{Data and Data Analysis}

The dataset used in this paper consists of observations in the B and V bands collected from the Cluster Ages Experiment (CASE) in the period June 1995-June 2009 (Kaluzny et al. 2013) and of I band observations from the M4 Core Project with the Hubble Space Telescope (HST) obtained between October 2012 and September 2013 (Nascimbeni et al. 2014). Period04 (Lenz \& Breger 2005) was used to determine the period of the variables. The epochs derived were used for period variation studies for the times of minima obtained from the CASE data (Kaluzny et al., 2013). These times were determined with a Gaussian fit to the observed eclipses in the data. In Fig. 1, we show the

*rukminiouastro@yahoo.com

†astroshanti@gmail.com 


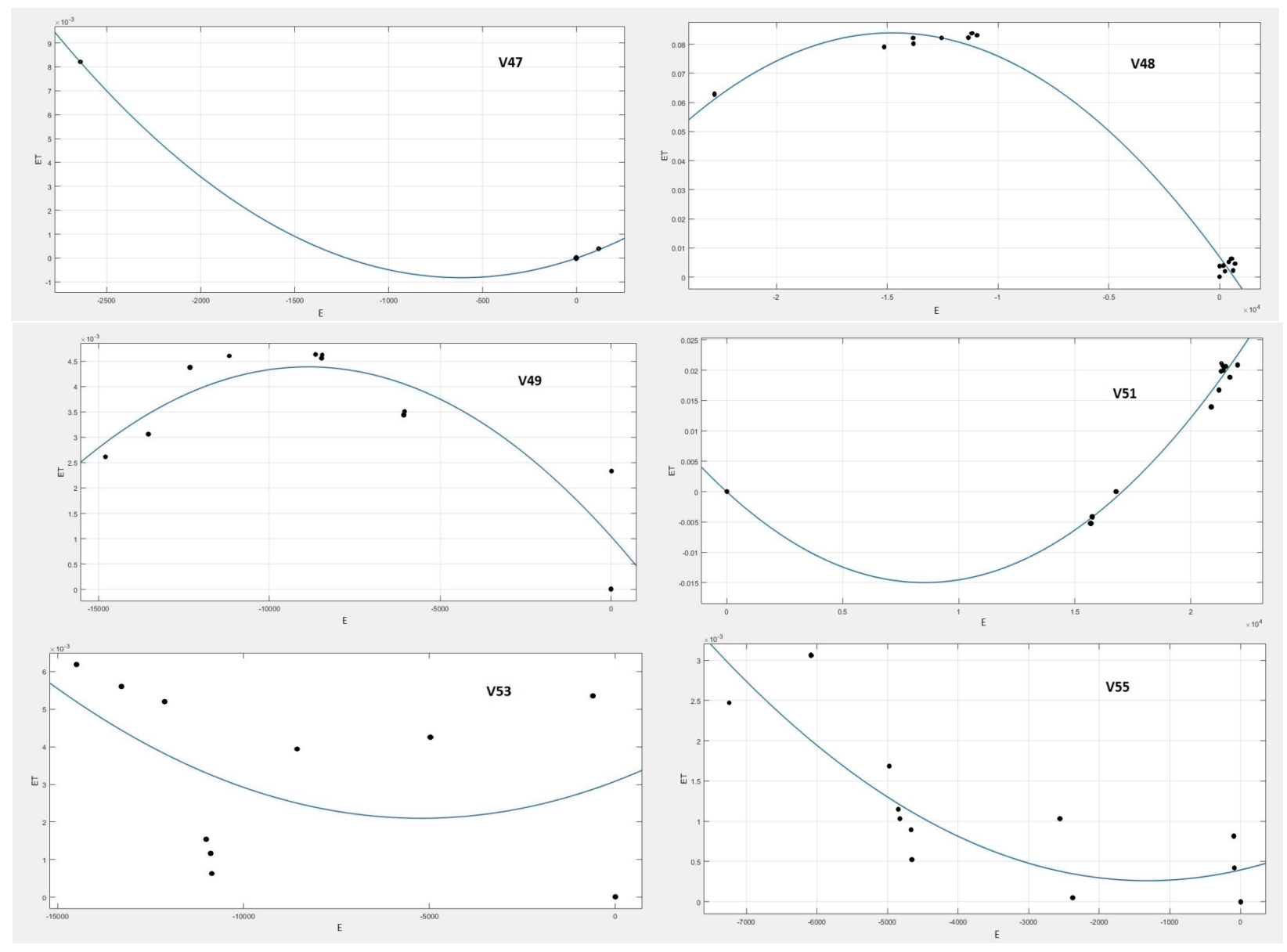

Figure 1: The eclipse timing diagrams for the variable stars V47 (top left), V48 (top right), V49 (middle left), V51 (middle right), V53 (bottom left), and V55 (bottom right). The observed eclipse timings and the best quadratic fit are given by black dots and a blue line, respectively. For each star, the best observed minimum was chosen as the zeropoint of the number of cycles E.

Table 1: The rate of period changes for the respective variables.

\begin{tabular}{cccc}
\hline S.No & ID & Period (days) & dp/dt (d/yr) \\
\hline 1 & V47 & 0.269873 & $5.94 \times 10^{-6}$ \\
2 & V48 & 0.282686 & $-9.14 \times 10^{-7}$ \\
3 & V49 & 0.297444 & $-1.04 \times 10^{-7}$ \\
4 & V51 & 0.303683 & $4.96 \times 10^{-7}$ \\
5 & V53 & 0.308448 & $8.54 \times 10^{-8}$ \\
6 & V55 & 0.310703 & $1.80 \times 10^{-7}$ \\
\hline
\end{tabular}



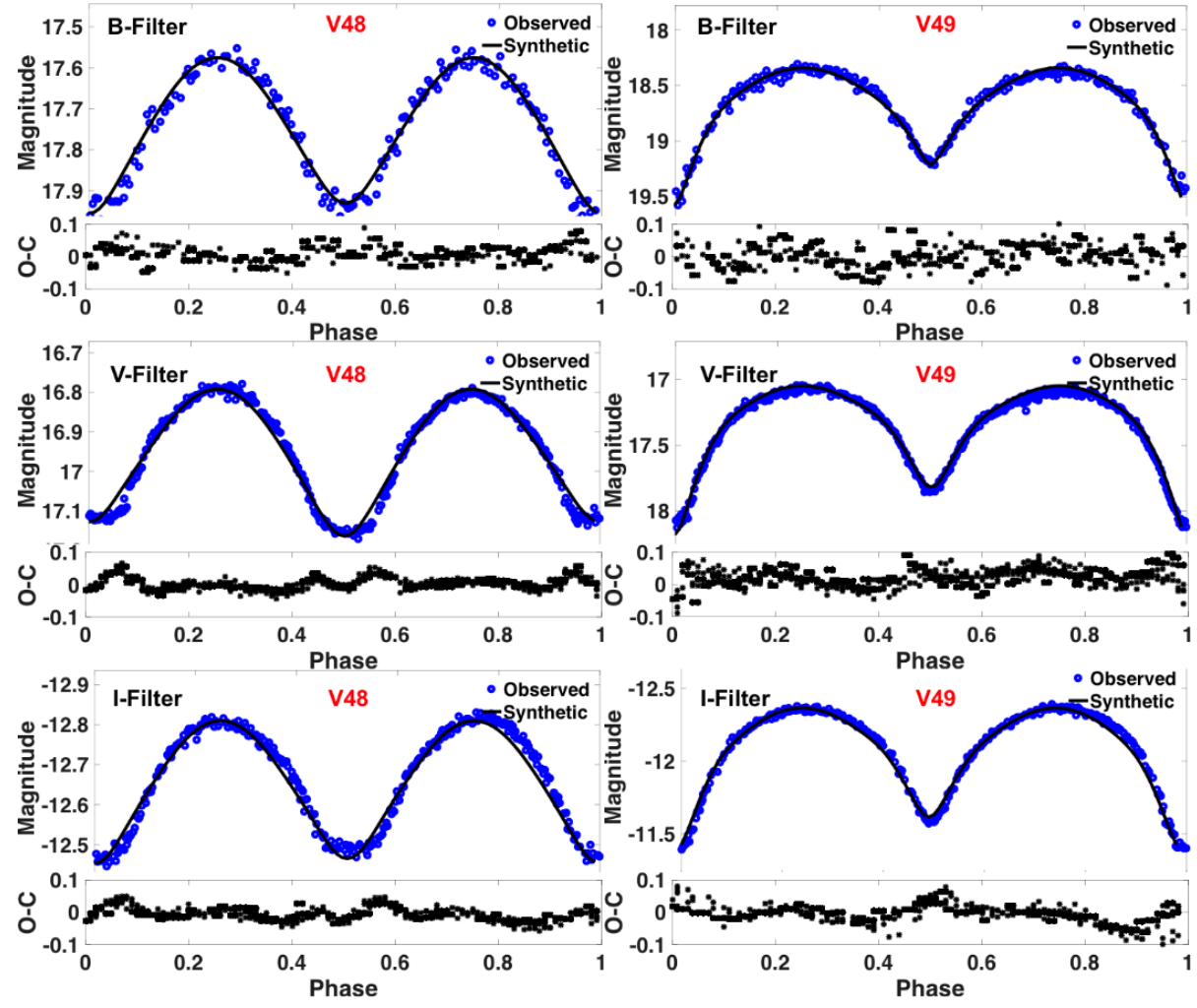

Figure 2: The best fit (full line) to the light curves (dots) for variables V48 (left) and V49 (right) in the B (top), V (middle), and I (bottom) band. The residuals are given in the bottom panels.
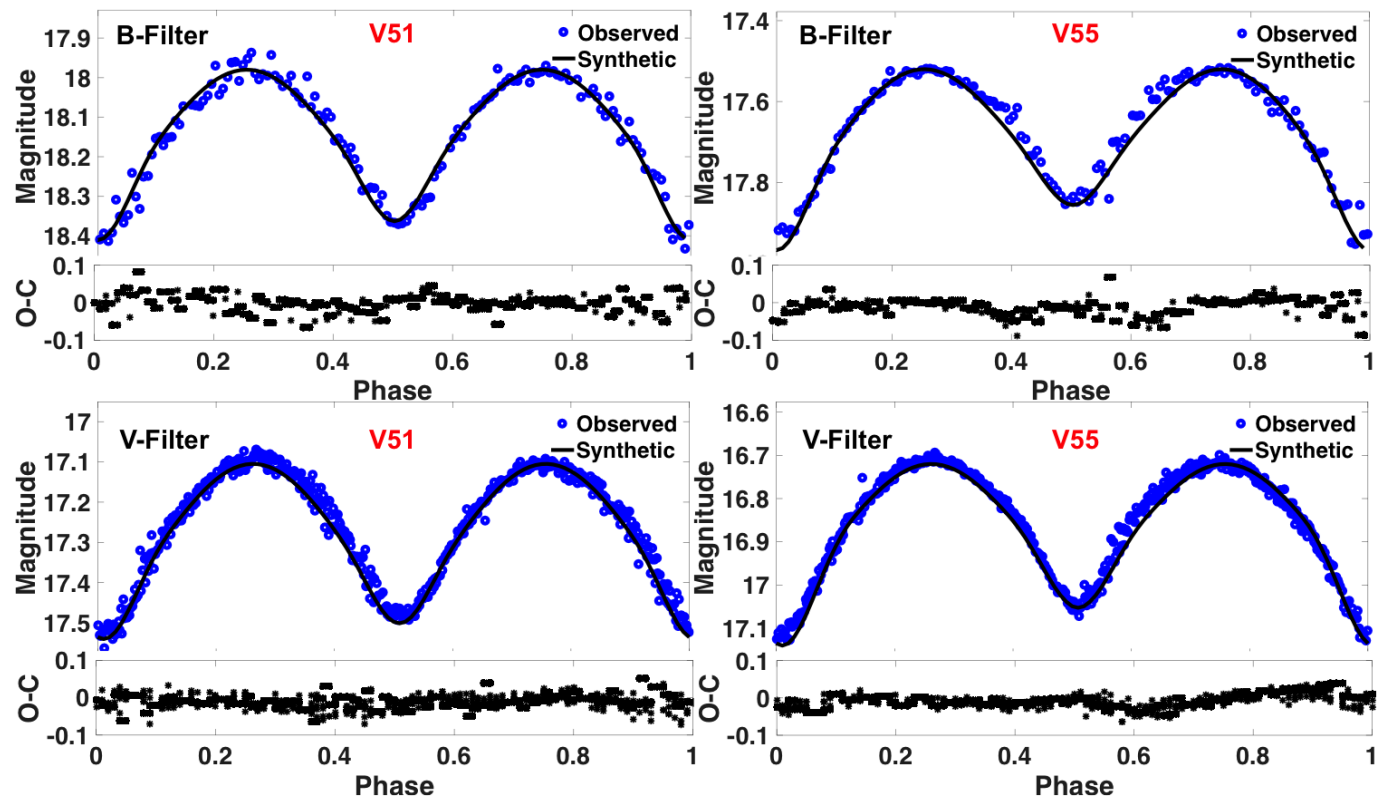

Figure 3: The best fit (full line) to the light curves (dots) for variables V51 (left) and V55 (right) in the B (top) and V (bottom) band. The residuals are given in the bottom panels. 
Table 2: Photometric solutions for the variables using WD code

\begin{tabular}{|c|c|c|c|c|c|}
\hline \multirow{2}{*}{$\begin{array}{l}\text { Photometric } \\
\text { Elements }\end{array}$} & \multicolumn{3}{|c|}{ V48 } & \multicolumn{2}{|c|}{ V51 } \\
\hline & B & V & I & B & V \\
\hline Period (d) & 0.282686 & 0.282686 & 0.282686 & 0.303683 & 0.303683 \\
\hline $\mathrm{T}_{e, h}(\mathrm{~K})$ & 6540 & 6540 & 6540 & 6250 & 6250 \\
\hline $\mathrm{T}_{e, c}(\mathrm{~K})$ & $6437 \pm 35$ & $6945 \pm 19$ & $6872 \pm 45$ & $6020 \pm 40$ & $6013 \pm 26$ \\
\hline q & $0.16 \pm 0.006$ & $0.1145 \pm 0.002$ & $0.124 \pm 0.003$ & $1.24 \pm 0.02$ & $1.37 \pm 0.02$ \\
\hline $\mathrm{i}^{o}$ & $80.03 \pm 2.07$ & $72.441 \pm 0.53$ & $77.704 \pm 1.12$ & $66.463 \pm 0.40$ & $66.28 \pm 0.23$ \\
\hline$\Omega$ & $2.07943 \pm 0.024$ & $1.95041 \pm 0.0087$ & $1.99121 \pm 0.0124$ & $4.07105 \pm 0.031$ & $4.24715 \pm 0.027$ \\
\hline fill-out factor & 0.4904 & 0.5446 & 0.3552 & 0.2690 & 0.2249 \\
\hline $\mathrm{r}_{h}$, pole & $0.5157 \pm 0.006$ & $0.5383 \pm 0.003$ & $0.5332 \pm 0.004$ & $0.3399 \pm 0.003$ & $0.3388 \pm 0.003$ \\
\hline $\mathrm{r}_{h}$, side & $0.5702 \pm 0.010$ & $0.6037 \pm 0.004$ & $0.5959 \pm 0.006$ & $0.3567 \pm 0.004$ & $0.3563 \pm 0.004$ \\
\hline $\mathrm{r}_{h}$, back & $0.5947 \pm 0.012$ & $0.6274 \pm 0.005$ & $0.6199 \pm 0.007$ & $0.3907 \pm 0.007$ & $0.3942 \pm 0.007$ \\
\hline $\mathrm{r}_{c}$, pole & $0.2311 \pm 0.014$ & $0.2196 \pm 0.008$ & $0.2225 \pm 0.010$ & $0.3810 \pm 0.003$ & $0.3935 \pm 0.003$ \\
\hline $\mathrm{r}_{c}$, side & $0.2423 \pm 0.017$ & $0.2313 \pm 0.010$ & $0.2340 \pm 0.012$ & $0.4023 \pm 0.004$ & $0.4172 \pm 0.004$ \\
\hline $\mathrm{r}_{c}$, back & $0.2905 \pm 0.042$ & $0.2943 \pm 0.035$ & $0.2928 \pm 0.040$ & $0.4342 \pm 0.006$ & $0.4515 \pm 0.005$ \\
\hline $\mathrm{L}_{h}$ & 0.8437 & 0.7958 & 0.8423 & 0.5254 & 0.4068 \\
\hline $\mathrm{L}_{c}$ & 0.1563 & 0.2042 & 0.1577 & 0.4746 & 0.5932 \\
\hline $\mathrm{x}_{h}$ & 0.6 & 0.6 & 0.6 & 0.6 & 0.6 \\
\hline $\mathrm{x}_{c}$ & 0.6 & 0.6 & 0.6 & 0.6 & 0.6 \\
\hline $\mathrm{W}(\mathrm{O}-\mathrm{C})^{2}$ & 0.0094 & 0.0059 & 0.0068 & 0.0173 & 0.1035 \\
\hline Spectral type & & & & F8 & F8 \\
\hline $\mathrm{A}_{h, c}$ & 1 & 1 & 1 & 1 & 1 \\
\hline $\mathrm{G}_{h, c}$ & 0.5 & 0.5 & 0.5 & 0.5 & 0.5 \\
\hline
\end{tabular}

\begin{tabular}{|l|ccc|cc|}
\hline Photometric & & $\mathrm{V} 49$ & & \multicolumn{2}{c|}{$\mathrm{V} 55$} \\
Elements & $\mathrm{B}$ & $\mathrm{V}$ & $\mathrm{I}$ & $\mathrm{B}$ & $\mathrm{V}$ \\
\hline Period $(\mathrm{d})$ & 0.297444 & 0.297444 & 0.297444 & 0.310703 & 0.310703 \\
$\mathrm{~T}_{e, h}(\mathrm{~K})$ & 4778 & 4778 & 4778 & 6555 & 6555 \\
$\mathrm{~T}_{e, c}(\mathrm{~K})$ & $4564 \pm 10$ & $4556 \pm 06$ & $4508 \pm 11$ & $5901 \pm 40$ & $6084 \pm 18$ \\
$\mathrm{q}$ & $1.05 \pm 0.05$ & $0.78 \pm 0.01$ & $0.806 \pm 0.02$ & $1.2 \pm 0.006$ & $1.09 \pm 0.007$ \\
$\mathrm{i}^{o}$ & $87.603 \pm 0.69$ & $88.068 \pm 0.66$ & $87.131 \pm 0.47$ & $65.244 \pm 0.40$ & $65.217 \pm 0.15$ \\
$\Omega$ & $3.65011 \pm 0.066$ & $3.30371 \pm 0.025$ & $3.2601 \pm 0.032$ & $4.03809 \pm 0.014$ & $3.88113 \pm 0.0108$ \\
fill-out factor & 0.0954 & 0.1504 & 0.3483 & 0.0542 & 0.0537 \\
$\mathrm{r}_{h}$, pole & $0.3785 \pm 0.01$ & $0.3925 \pm 0.004$ & $0.3982 \pm 0.005$ & $0.3453 \pm 0.001$ & $0.3495 \pm 0.001$ \\
$\mathrm{r}_{h}$, side & $0.4019 \pm 0.01$ & $0.4165 \pm 0.005$ & $0.4245 \pm 0.007$ & $0.3626 \pm 0.002$ & $0.3668 \pm 0.001$ \\
$\mathrm{r}_{h}$, back & $0.4463 \pm 0.02$ & $0.4534 \pm 0.008$ & $0.4681 \pm 0.013$ & $0.3961 \pm 0.003$ & $0.3983 \pm 0.002$ \\
$\mathrm{r}_{c}$, pole & $0.3783 \pm 0.01$ & $0.3480 \pm 0.004$ & $0.3625 \pm 0.006$ & $0.3748 \pm 0.001$ & $0.3639 \pm 0.001$ \\
$\mathrm{r}_{c}$, side & $0.4017 \pm 0.01$ & $0.3669 \pm 0.005$ & $0.3844 \pm 0.008$ & $0.3953 \pm 0.002$ & $0.3827 \pm 0.001$ \\
$\mathrm{r}_{c}$, back & $0.4463 \pm 0.02$ & $0.4073 \pm 0.009$ & $0.4325 \pm 0.015$ & $0.4274 \pm 0.003$ & $0.4136 \pm 0.002$ \\
$\mathrm{~L}_{h}$ & 0.5856 & 0.6966 & 0.5981 & 0.6498 & 0.5726 \\
$\mathrm{~L}_{c}$ & 0.4144 & 0.3034 & 0.4019 & 0.3502 & 0.4274 \\
$\mathrm{x}_{h}$ & 0.6 & 0.6 & 0.6 & 0.6 & 0.6 \\
$\mathrm{x}_{c}$ & 0.6 & 0.6 & 0.6 & 0.6 & 0.6 \\
$\mathrm{~W}(\mathrm{O}-\mathrm{C})^{2}$ & 0.0503 & 0.079 & 0.029 & 0.015 & 0.0417 \\
Spectral type & $\mathrm{K} 2-3$ & $\mathrm{~K} 2-3$ & & $\mathrm{~F} 6$ & $\mathrm{~F} 6$ \\
$\mathrm{~A}_{h, c}$ & 0.5 & 0.5 & 0.5 & 1 & 1 \\
$\mathrm{G}_{h, c}$ & 0.32 & 0.32 & 0.32 & 0.5 & 0.5 \\
\hline
\end{tabular}


eclipse timing (ET) diagrams: the differences between the observed and computed times of minima are shown as a function of the cycle number E.

The best quadratic fits to the observed eclipse timings were obtained for all the variables. The resulting equations are as follows:

$$
\begin{aligned}
& \mathrm{ET}_{V 47}=2.193( \pm 0.042) \times 10^{-9} \times \mathrm{E}^{2}+2.686( \pm 0.001) \times 10^{-6} \times \mathrm{E}-0.00006( \pm 0.0008) \\
& \mathrm{ET}_{V 48}=-3.536( \pm 1.895) \times 10^{-10} \times \mathrm{E}^{2}-1.044( \pm 1.122) \times 10^{-5} \times \mathrm{E}+0.006874( \pm 0.00423) \\
& \mathrm{ET}_{V 49}=-4.252( \pm 1.831) \times 10^{-11} \times \mathrm{E}^{2}-7.542( \pm 0.109) \times 10^{-7} \times \mathrm{E}+0.001043( \pm 0.00013) \\
& \mathrm{ET}_{V 51}=2.063( \pm 1.549) \times 10^{-10} \times \mathrm{E}^{2}-3.515( \pm 1.003) \times 10^{-6} \times \mathrm{E}-0.0000217( \pm 0.00003) \\
& \mathrm{ET}_{V 53}=3.606( \pm 2.522) \times 10^{-11} \times \mathrm{E}^{2}+3.770( \pm 0.106) \times 10^{-7} \times \mathrm{E}+0.00308( \pm 0.0014) \\
& \mathrm{ET}_{V 55}=7.672( \pm 0.736) \times 10^{-11} \times \mathrm{E}^{2}+2.025( \pm 1.642) \times 10^{-7} \times \mathrm{E}+0.000395( \pm 0.00012)
\end{aligned}
$$

The rates of period change were computed from the best fit quadratic equations and are listed in the Table 1. The photometric solutions were obtained using the differential correction code and light curve synthesis code (DC \& LC) of the WD code (Wilson \& Devinney 1971; Van Hamme \& Wilson 2003) for the variables V48, V49, V51, V55 and the derived elements are shown in the Table 2 for the data in different passbands. For the meaning of different parameters listed in Table 2, we refer to Shanti Priya et al. (2013). Note that V47 and V53 were omitted because they are well studied in the literature. The observed and theoretical light curve fits are shown along with the residuals in the top and bottom panels, respectively, of Fig. 2 and Fig. 3.

\section{Discussion and Conclusions}

The WD code (v2003) was used to derive the fundamental parameters of contact binaries in M4. The theoretical and observed light curves for four variables in different photometric filters are plotted. From the observed temperature difference between the components and the fill out factors derived for these systems, it is concluded that all the binaries are in good geometrical and thermal contact except for variable V55. The eclipse timing diagrams for all the six variables reveal a secular change in their periods, with an increasing rate for all except for V47 and V49. The rate of period changes determined for the variables in the cluster M4 are found to be similar to that observed for the field stars. The secular change could be due to the presence of a tertiary component or to mass transfer or mass loss in the system (Zhang et al. 2016, Erkan\& Ulas 2016). This can be evaluated through long term variation studies using planned observations with 3.6-m DOT and 4.0-m ILMT at Devasthal Observatory.

\section{References}

Bassa C., Pooley D., Homer L. et al. 2004, ApJ, 609, 755

Erkan N., Ulas B. 2016, NewA, 46,73

Kaluzny J., Thompson I.B., Rozyczka M., Krzeminski W. 2013, AcA, 63, 181

Lenz P., Breger M. 2005, CoAst 146, 53

Nascimbeni V., Bedin L. R., Heggie D. C. et al. 2014, MNRAS, 442, 2381

Shanti Priya D. 2013, RAA, 13, 465

Van Hamme W., Wilson R. E. 2003, ASPC, 298, 323

Wilson R. E., Devinney E. J. 1971, ApJ, 166, 605

Zang Y., Han Q. W., Liu J. Z. 2016, PASP, 128,124201 